\title{
TWO NEW DIFFERENTIAL EQUATIONS OF TURBULENT DISSIPATION RATE AND APPARENT VISCOSITY FOR NON-NEWTONIAN FLUIDS
}

\author{
Fabio Goria , Andrea Boghib \\ a, ASME Fellow and Corresponding Author, fammannati@yahoo.com, \\ ${ }^{a, b}$ Department of Mechanical Engineering, University of Rome “Tor Vergata”, 00133 Rome, Italy.
}

\begin{abstract}
A new equation for the dissipation rate of turbulent kinetic energy is derived exactly in conservative form for a Generalized Newtonian Fluid (GNF). The transport equations for mass, momentum, and turbulent kinetic energy are written along to the transport equation for the shear rate. A new transport equation for the apparent viscosity is derived assuming the viscosity as dependent only on the shear rate. The assumption is of incompressible two-dimensional GNF flow.
\end{abstract}

Keywords: Apparent viscosity, Dissipation rate, Turbulent energy, Shear rate, Generalized Newtonian Fluid.

\section{Introduction}

Non-Newtonian fluids are present in several industrial applications and biological problems, like blood flow. Literature presents many theoretical solutions and numerical simulations in laminar flow, including two papers published by the first author more than thirty years ago [1-2].

Few numerical investigations dealt with turbulent flow of pseudo-plastic fluids (shear-thinning fluid) because of the lack of models with one or two point closure and, for this reason, some investigators performed DNS (Direct Numerical Simulation). Rudman and Blackburn used the Spectral Element-Fourier Method (SEM) in a duct flow, [3], and compared the DNS results of a power law fluid with small consistency index and a Herschel-Bulkley fluid with experimental data [4]. Dimitropoulos [5-6] carried out DNS of a polymeric solution using FENE-P and Giesekus models, employing spectral approximation and semi-implicit algorithm to predict the drag reduction. New results on Reynolds stresses and pressure are presented in [6], where the convergence of the pseudo-spectral algorithm is discussed. A non-refined mesh and a high artificial viscosity are introduced to stabilize the algorithm. The FENE-P model is used in [7] for a DNS one-dimensional approach to explain the phenomenon of drag reduction. A turbulent model for a non-Newtonian power law fluid is developed in [8], in analogy to the turbulent viscosity, determining the temperature distribution for soybean milk flowing inside a tubular heat exchanger.

Turbulent flow of a non-Newtonian fluid is important also in the medical field. A model to predict the turbulent flow of a power-law fluid in a bio-reactor for anaerobic digestion is developed in [9] with the classical $k-\varepsilon$ model and the power-law viscosity. The $k-\varepsilon$ equations are derived in [10-11] for power-law and Herschel-Bulkley fluid using the apparent viscosity of a non-Newtonian fluid in the RANS equations of a Newtonian fluid, but the agreement is not good enough. The introduction of the third invariant of the rate of deformation tensor in the viscosity contributes to an increase of viscous diffusion and dissipation rate in the turbulent kinetic energy confirming the dependence of the viscosity on the second invariant of the rate of deformation tensor in a 2D flow [12]. The Generalized Newtonian Fluid (GNF) constitutive equation is applied to a Bird-Carreau fluid in order to derive a $k-\varepsilon$ model for the equations of Reynolds stresses tensor, 
turbulent kinetic energy and dissipation rate [13]. The viscosity is dependent on the invariants of the rate of deformation tensor, shear-rate and strain-rate. An algebraic equation is proposed to correlate the instantaneous viscosity to the dissipation rate while average viscosity and dissipation rate are correlated with a normal logarithmic probability distribution of the dissipation rate. The final equation of dissipation rate is written in non conservative form because two derivatives are present, one for the dissipation rate itself and the other for the average dynamic viscosity.

Direct Numerical Simulation of viscoelastic fluids in turbulent channel flow is carried out using the FENE-P model to find relationships between flow and fluid rheological parameters [14]. Three different regimes of drag reduction, namely low, high and medium are identified proposing mathematical expressions for the eddy viscosity in the three regimes. A procedure based on the DNS predictions of the budgets of momentum and viscoelastic shear stress is developed to evaluate the mean velocity profile. A RANS model is employed using the FENE-P constitutive relationship to describe the rheology of polymer-induced turbulent drag reduction [15]. Correlations among flow and polymer conformation variables are identified by analyzing recent DNS results of dilute polymer solutions.

The present work is aimed to derive the equation of the dissipation rate in a conservative form for an incompressible GNF in 2D (two-dimensional) flow. Viscosity is on the shear-rate, as shown in [12] in a 2D flow and done in all the papers found in the literature, without an explicit statement of the relation with the shear-rate. A 2D theory of turbulence has been developed since the 70's in [16-20] despite the differences with the real 3D flow and the absence of vortex-stretching terms. It is also affirmed in [21] that it seems natural to investigate the behavior of a $2 \mathrm{D}$ flow, in the hope that it sheds some light on "almost" $2 \mathrm{D}$ turbulence. Due to the difficulty of deriving the governing equations directly in a 3D flow, it is considered a guide to develop first a 2D turbulent model. The importance of studying a 2D model, which can be generalized in 3D, is also remarked in [12], where the authors developed an order of magnitude analysis for a 2D turbulent flow, without loss of generality.

The transport equation of $\varepsilon$ is deduced in this work by the use of a completely new transport equation for the apparent viscosity and without a constitutive link between apparent viscosity and shear rate. No hypotheses are necessary on the dependence of the turbulent dissipation rate on the fluctuating part of the rate of deformation tensor, as required in [15], neither a particular statistical distribution of the average viscosity, as required in [16]. The present procedure allows to obtain a new transport equation of $\varepsilon$ in a conservative form, more general than that obtained previously in the literature. Moreover, the conservative form of the $\varepsilon$ equation allows to avoid the calculation of the temporal derivative of the apparent viscosity, due to the presence of a transport equation for the apparent viscosity.

The paper has the following structure. The transport equations for the average variables and the turbulent kinetic energy are derived first and then the transport equation for the shear-rate. The new differential equation for the apparent viscosity is deduced using the scalar product of the instantaneous rate of deformation tensor by itself. From this equation it is possible to derive the equation of dissipation rate in a conservative form and to give a physical interpretation to the new terms. The method used in this work 
allows to explain each term and to classify as transport, production and dissipation terms. Finally, an order of magnitude analysis is carried out.

\section{Nomenclature}

\section{Latin}

$k \quad$ mean turbulent kinetic energy

$k^{\prime} \quad$ instantaneous turbulent kinetic energy

$l \quad$ viscous length scale,

$L \quad$ geometric length scale,

$p \quad$ instantaneous static pressure

$P \quad$ mean static pressure

$p^{\prime} \quad$ fluctuating static pressure

$S \quad$ shear rate

$S_{i j} \quad$ rate of deformation tensor

$\left\langle S_{i j}\right\rangle \quad$ mean rate of deformation tensor

$S_{i j}^{\prime} \quad$ fluctuating rate of deformation tensor

$\$ \quad$ variable defined in Eq. 20

$T_{R, i j} \quad$ mean Reynolds stress tensor

$T_{\mu, i j} \quad$ mean polymeric stress tensor

$T_{R, i j}^{\prime} \quad$ instantaneous Reynolds stress tensor

$T_{\mu, i j}^{\prime} \quad$ instantaneous polymeric stress tensor

$t \quad$ time

$u_{i} \quad$ instantaneous i-velocity component

$U_{i} \quad$ mean $\mathrm{x}$ i-velocity component

$x_{i} \quad i$ - coordinate

\section{Greek}

$\dot{\gamma} \quad$ shear rate

$\delta_{i j} \quad$ Kronecher delta

$\varepsilon \quad$ mean dissipation rate

$\varepsilon^{\prime} \quad$ instantaneous dissipation rate

$\lambda$ Taylor length scale

$\lambda_{\mu} \quad$ viscosity fluctuation length scale

$\mu_{a p p} \quad$ apparent viscosity 
$\left\langle\mu_{\text {app }}\right\rangle$ mean apparent viscosity

$\mu_{a p p}^{\prime} \quad$ fluctuating apparent viscosity

$\rho$ density

$\tau_{c} \quad$ yield stress

$\Omega_{i j} \quad$ rotation rate tensor

$\left\langle\Omega_{i j}\right\rangle$ mean rotation rate tensor

$\Omega_{i j}^{\prime} \quad$ fluctuating rotation rate tensor

\section{Constitutive equation}

The present 2D analysis is carried on for a Generalized Newtonian Fluid, GNF. The constitutive equation for an incompressible non-Newtonian fluid is written similarly to a Newtonian one with the apparent viscosity function of the shear-rate

$T_{i j}=-p \delta_{i j}+2 \mu_{a p p} S_{i j}$,

where $T_{i j}$ is the stress tensor and $p$ the static pressure.

The rate of deformation tensor $S_{i j}$ is

$$
S_{i j}=\frac{1}{2}\left(\frac{\partial u_{i}}{\partial x_{j}}+\frac{\partial u_{j}}{\partial x_{i}}\right),
$$

and the shear-rate $\dot{\gamma}$ is

$\dot{\gamma}=\sqrt{2 S_{i j}^{2}}$.

Defining $S$ as

$S=\frac{\dot{\gamma}}{\sqrt{2}}$,

the shear-rate will be treated as $S$ from now on.

\section{Conservation equations of mass, momentum and turbulent kinetic energy}

The conservation equations for the instantaneous variables in a 2D flow are the followings:

$\frac{\partial u_{i}}{\partial x_{i}}=0$

for the mass, and

$\rho \frac{\partial u_{i}}{\partial t}+\rho u_{k} \frac{\partial u_{i}}{\partial x_{k}}=-\frac{\partial p}{\partial x_{i}}+\frac{\partial}{\partial x_{k}}\left(2 \mu_{a p p} S_{i k}\right)$,

for the momentum.

Each instantaneous variable is decomposed into a mean and a fluctuating component

$u_{i}=U_{i}+u_{i}^{\prime}$, 
$p=P+p^{\prime}$

$\mu_{a p p}=\left\langle\mu_{a p p}\right\rangle+\mu_{a p p}^{\prime}$.

The mean component of the stress tensor, Eq. (1), becomes

$$
\left\langle T_{i j}\right\rangle=-P \delta_{i j}+2\left\langle\mu_{a p p}\right\rangle\left\langle S_{i j}\right\rangle+2\left\langle\mu_{a p p}^{\prime} S_{i j}^{\prime}\right\rangle,
$$

and the fluctuating one

$T_{i j}^{\prime}=-p^{\prime} \delta_{i j}+2 \mu_{a p p}^{\prime}\left\langle S_{i j}\right\rangle+2\left\langle\mu_{a p p}\right\rangle S_{i j}^{\prime}+2 \mu_{a p p}^{\prime} S_{i j}^{\prime}-2\left\langle\mu_{a p p}^{\prime} S_{i j}^{\prime}\right\rangle$.

The third term of the mean component, Eq. (10), is due to the viscosity fluctuations while different combinations of mean and fluctuating components are present in the fluctuating tensor, Eq. (11).

The conservation equations of the mean variables are

$$
\frac{\partial U_{i}}{\partial x_{i}}=0
$$

for the mass, and

$\rho \frac{\partial U_{i}}{\partial t}+\rho U_{k} \frac{\partial U_{i}}{\partial x_{k}}=-\frac{\partial P}{\partial x_{i}}+\frac{\partial}{\partial x_{k}}\left(2\left\langle\mu_{a p p}\right\rangle\left\langle S_{i k}\right\rangle+T_{R, i j}+T_{\mu, i j}\right)$,

for the momentum.

In the mean flow, the Reynolds stress tensor is given by

$$
T_{R, i j}=-\left\langle\rho u_{i}^{\prime} u_{j}^{\prime}\right\rangle \text {, }
$$

while the polymeric stress tensor, so-called also for a non-polymer fluid, is, according to [13],

$T_{\mu, i j}=2\left\langle\mu_{a p p}^{\prime} S_{i j}^{\prime}\right\rangle$.

For constant viscosity the Reynolds stresses, due to convection, are the only terms responsible for the energy transfer from mean to fluctuating scale, while, for variable viscosity, the polymeric stresses are active in conjunction to the Reynolds stresses in the energy transfer from mean to fluctuating scale.

The final equation for the turbulent kinetic energy is

$$
\begin{aligned}
& \frac{\partial}{\partial t}(\rho k)+U_{j} \frac{\partial}{\partial x_{j}}(\rho k)=\left(T_{R, i j}-T_{\mu, i j}\right)\left\langle S_{i j}\right\rangle+ \\
& -\frac{\partial}{\partial x_{j}}\left(\left\langle\rho k^{\prime} u_{j}^{\prime}\right\rangle+\left\langle p^{\prime} u_{j}^{\prime}\right\rangle-\left\langle\mu_{a p p}^{\prime} \frac{\partial k^{\prime}}{\partial x_{j}}\right\rangle-\left\langle\mu_{a p p}\right\rangle \frac{\partial k}{\partial x_{j}}-2\left\langle u_{i}^{\prime} \mu_{a p p}^{\prime}\right\rangle\left\langle S_{i j}\right\rangle-\frac{\left\langle\mu_{a p p}\right\rangle}{\rho} \frac{\partial T_{R, i j}}{\partial x_{i}}-\left\langle\frac{\mu_{a p p}^{\prime}}{\rho} \frac{\partial T_{R, i j}^{\prime}}{\partial x_{i}}\right\rangle\right)+ \\
& -2\left\langle\mu_{a p p}\right\rangle\left\langle S_{i j}^{\prime} S_{i j}^{\prime}\right\rangle-2\left\langle\mu_{a p p}^{\prime} S_{i j}^{\prime} S_{i j}^{\prime}\right\rangle
\end{aligned}
$$

where the groups of terms on the right hand side are given by 


$$
\begin{aligned}
& P^{k}=\left(T_{R, i j}-T_{\mu, i j}\right)\left\langle S_{i j}\right\rangle \\
& \Pi^{k}=-\frac{\partial}{\partial x_{j}}\left(\left\langle p^{\prime} u_{j}^{\prime}\right\rangle\right) \\
& T^{k}=-\frac{\partial}{\partial x_{j}}\left(\left\langle\rho k^{\prime} u_{j}^{\prime}\right\rangle\right) \\
& D^{k}=\frac{\partial}{\partial x_{j}}\left(\left\langle\mu_{a p p}\right\rangle \frac{\partial k}{\partial x_{j}}+\frac{\left\langle\mu_{a p p}\right\rangle}{\rho} \frac{\partial T_{R, i j}}{\partial x_{i}}+\left\langle\frac{\mu_{a p p}^{\prime}}{\rho} \frac{\partial T_{R, i j}^{\prime}}{\partial x_{i}}\right\rangle+\left\langle\mu_{a p p}^{\prime} \frac{\partial k^{\prime}}{\partial x_{j}}\right\rangle+2\left\langle u_{i}^{\prime} \mu_{a p p}^{\prime}\right\rangle\left\langle S_{i j}\right\rangle\right)_{, j} \\
& \varepsilon=2\left\langle\mu_{a p p}\right\rangle\left\langle S_{i j}^{\prime} S_{i j}^{\prime}\right\rangle+2\left\langle\mu_{a p p}^{\prime} S_{i j}^{\prime} S_{i j}^{\prime}\right\rangle
\end{aligned}
$$

\section{Discussion on the turbulent kinetic energy}

The first group, $P^{k}$, is a production one. In a Newtonian fluid only the term due to the product of Reynolds stresses and mean strain rates is present while in a GNF flow is present also a term which takes into account the polymeric stresses, with an opposite sign. In the mean equation, Eq. (10), the Reynolds stresses component is added to the polymeric stresses while in Eq. (16) they subtract because have a different influence on the fluid motion. If the Reynolds stresses transfer energy from the mean flow to increase the fluctuating component, the polymeric stresses will reduce these effects. In conclusion, the Reynolds stresses transfer energy from the mean flow to increase the fluctuating component while the polymeric stresses reduce the effects of the Reynolds stresses.

The groups, $\Pi^{k}+T^{k}+D^{k}$, are transport ones. The two groups $\Pi^{k}+T^{k}$ and the first two terms of the group $D^{k}$ are present also in a Newtonian fluid while the remaining three terms of $D^{k}$, which are the mean product of instantaneous viscosity and each instantaneous variable, are present only in a GNF flow. The last term, i.e. the product of the mean rate of deformation tensor, the mean product of the velocity fluctuation and the apparent viscosity fluctuation, has a diffusive form, as the others present in the divergence operator, and is directly connected to the mean flow because is dependent on the mean rate of deformation tensor.

The last group, $\mathcal{E}$, is the dissipation one. Two terms are present because viscosity is split into a mean and a fluctuating component. The first term is the product of the mean viscosity and the mean square of the fluctuating shear rate while the second one is the mean of the product of the fluctuating viscosity and the square of the fluctuating shear rate. A term can be classified productive if positive, or if contributes to the increase of a certain variable, while is dissipative in the opposite case. In the second term, the viscosity fluctuations are unknown, a priori, as concurrent or not to the velocity fluctuations gradient and cannot be stated if these terms are productive or dissipative from a theoretical point of view. The square of the fluctuating strain rates is positive but cannot be said a priori if it is a dissipative term when is multiplied for the viscosity fluctuations and averaged. The choice to call this term productive or dissipative depends on the similarity with the classical production or dissipation term. In conclusion, it is possible to consider the term with the fluctuating viscosity as an "increase" or a "decrease" of the production or an "increase" or a "decrease" of the dissipation.

\section{Transport equations for mean and fluctuating shear rate.}


The equation for the dissipation rate, $\varepsilon$, of the turbulent kinetic energy is written in conservative form as $\rho \frac{D \varepsilon}{D t}=f\left(x_{j}, t\right)$

being defined, in analogy to [13], as

$\varepsilon=\frac{2}{\rho}\left\langle\mu_{a p p}\right\rangle\left\langle \$^{2}\right\rangle+\frac{2}{\rho}\left\langle\mu_{a p p}^{\prime} \$^{2}\right\rangle$

where

$\$^{2}=S_{i j}^{\prime} S_{i j}^{\prime}$.

The transport equation for the rate of deformation tensor, $S_{i j}$, is obtained considering the symmetrical part of the gradient of Eq. (6). Using the definition of $\Omega_{i j}$

$$
\Omega_{i j}=\frac{1}{2}\left(\frac{\partial u_{i}}{\partial x_{j}}-\frac{\partial u_{j}}{\partial x_{i}}\right),
$$

the transport equations for the $S_{i j}$ components are

$$
\begin{aligned}
& \rho \frac{\partial S_{i j}}{\partial t}+\rho u_{k} \frac{\partial S_{i j}}{\partial x_{k}}=-\frac{1}{2} \frac{\partial}{\partial x_{i}}\left(\frac{\partial p}{\partial x_{j}}\right)-\frac{1}{2} \frac{\partial}{\partial x_{j}}\left(\frac{\partial p}{\partial x_{i}}\right)+\frac{\partial}{\partial x_{k}}\left(\mu_{a p p} \frac{\partial S_{i j}}{\partial x_{k}}+S_{i j} \frac{\partial \mu_{a p p}}{\partial x_{k}}\right)+ \\
& \left(-\rho S_{j k}^{2}+\rho \Omega_{j k}^{2}+\frac{\partial}{\partial x_{k}}\left(\left(S_{k j}-\Omega_{k j}\right) \frac{\partial \mu_{a p p}}{\partial x_{j}}\right)\right) \delta_{i j}
\end{aligned}
$$

The transport equation for the square of the shear rate, $S^{2}$, is obtained multiplying Eq. (22) for each term of the rate of deformation tensor by itself and summing all of them.

$$
\begin{aligned}
& \rho \frac{\partial}{\partial t}\left(\frac{S^{2}}{2}\right)+\rho u_{k} \frac{\partial}{\partial x_{k}}\left(\frac{S^{2}}{2}\right)=\frac{\partial}{\partial x_{k}}\left(\mu_{a p p} \frac{\partial}{\partial x_{k}}\left(\frac{S^{2}}{2}\right)+S^{2} \frac{\partial \mu_{a p p}}{\partial x_{k}}-S_{k j} \frac{\partial p}{\partial x_{j}}\right)+ \\
& -\left(\frac{\partial}{\partial x_{k}}\left(\mu_{a p p} S_{i j}\right)\right) \frac{\partial S_{i j}}{\partial x_{k}}+\frac{\partial S_{k j}}{\partial x_{k}} \frac{\partial p}{\partial x_{k}}
\end{aligned}
$$

A transport equation for the fluctuating components of the rate of deformation tensor is obtained by subtracting the mean equation from the instantaneous one

$$
\begin{aligned}
& \rho \frac{\partial S_{i j}^{\prime}}{\partial t}+\rho U_{k} \frac{\partial S_{i j}^{\prime}}{\partial x_{k}}=-\rho u_{k}^{\prime} \frac{\partial\left\langle S_{i j}\right\rangle}{\partial x_{k}}-\frac{1}{2} \frac{\partial}{\partial x_{i}}\left(\frac{\partial p^{\prime}}{\partial x_{j}}\right)-\frac{1}{2} \frac{\partial}{\partial x_{j}}\left(\frac{\partial p^{\prime}}{\partial x_{i}}\right)+ \\
& \frac{\partial}{\partial x_{k}}\left(-\rho u_{k}^{\prime} S_{i j}^{\prime}+\left\langle\rho u_{k}^{\prime} S_{i j}^{\prime}\right\rangle-\frac{\partial}{\partial x_{k}}\left(\left\langle\mu_{a p p}^{\prime} S_{i j}^{\prime}\right\rangle\right)+\frac{\partial}{\partial x_{k}}\left(\mu_{a p p} S_{i j}^{\prime}\right)+\frac{\partial}{\partial x_{k}}\left(\mu_{a p p}^{\prime}\left\langle S_{i j}\right\rangle\right)+\right. \\
& \left(-2 \rho S_{j k}^{\prime}\left\langle S_{j k}\right\rangle+2 \rho \Omega_{j k}^{\prime}\left\langle\Omega_{j k}\right\rangle-\rho S_{j k}^{\prime} S_{j k}^{\prime}+\rho \Omega_{j k}^{\prime} \Omega_{j k}^{\prime}\right) \delta_{i j}+ \\
& \left(\frac{\partial}{\partial x_{k}}\left(-\left\langle\left(S_{k j}^{\prime}-\Omega_{k j}^{\prime}\right) \frac{\partial \mu_{a p p}^{\prime}}{\partial x_{j}}\right\rangle+\left(S_{k j}^{\prime}-\Omega_{k j}^{\prime}\right) \frac{\partial \mu_{a p p}}{\partial x_{j}}+\left(\left\langle S_{k j}\right\rangle-\left\langle\Omega_{k j}\right\rangle\right) \frac{\partial \mu_{a p p}^{\prime}}{\partial x_{j}}\right)\right) \delta_{i j}
\end{aligned}
$$


The conservation equation for the dissipative terms of the turbulent kinetic energy is written using the conservation equation for the variable $\$^{2}$, obtained multiplying the transport equation of each term for the rate of deformation tensor, Eq. (24), by itself and summing all of them. The following expression is finally obtained

$$
\begin{aligned}
& \rho \frac{D}{D t}\left(\frac{\$^{2}}{2}\right)=\frac{1}{2} \nabla\left[T_{R}^{\prime}\right]: \nabla\langle[S]\rangle-\rho \nabla k^{\prime} \cdot(\nabla \cdot\langle[S]\rangle)-\nabla\left(\mu_{a p p}^{\prime}\langle[S]\rangle\right) \vdots \nabla\left[S^{\prime}\right]+ \\
& \nabla \cdot\left(-\left[S^{\prime}\right] \nabla p^{\prime}-\rho \vec{u}^{\prime} \frac{\$^{2}}{2}+\mu_{a p p} \nabla\left(\frac{\$^{2}}{2}\right)+\$^{2} \nabla \mu_{a p p}+\left[S^{\prime}\right]: \nabla\left(\mu_{a p p}^{\prime}\langle[S]\rangle\right)+\left[S^{\prime}\right] \rho \nabla k-\frac{1}{2}\left[S^{\prime}\right]: \nabla\left(\left[T_{R}\right]+\left[T_{\mu}\right]\right)\right)+ \\
& \nabla p^{\prime} \cdot\left(\nabla \cdot\left[S^{\prime}\right]\right)-\nabla\left(\mu_{a p p}\left[S^{\prime}\right]\right) \vdots \nabla\left[S^{\prime}\right]+\frac{1}{2} \nabla\left(\left[T_{R}\right]+\left[T_{\mu}\right]\right): \nabla\left[S^{\prime}\right]-\rho \nabla k \cdot\left(\nabla \cdot\left[S^{\prime}\right]\right)
\end{aligned}
$$

The conservation equation for the dissipation rate can be obtained by Eq. (19), using Eq. (25), and applying the principle of conservation. This operation is not trivial because viscosity is variable. For a Newtonian fluid the conservation equations can be obtained easily because each one has the same structure. Multiplication of each variable for the relative transport equation of the other and summation are necessary to derive the transport equation for the product of the two terms.

\section{Transport equation for the apparent viscosity}

In order to obtain the conservation equations for the turbulent dissipation rate the following equations are necessary:

- Transport equation of the apparent viscosity.

- Transport equation of the apparent viscosity, written to recognize terms similar to $\$^{2}$.

- Transport equation of the apparent viscosity multiplied for the transported term and summed to the product of the transport equation with the viscosity.

The apparent viscosity of a Newtonian fluid is a physical property while in a non-Newtonian ReinerRivlin fluid is function of the shear rate and can be generally written as function of $S$, on his side function of space and time, as

$\mu_{a p p}=f\left(S\left(x_{j}, t\right)\right)$.

Taking into account the following relations

$$
\frac{\partial \mu_{a p p}}{\partial t}=\frac{d f}{d S} \frac{\partial S}{\partial t}, \frac{\partial \mu_{a p p}}{\partial x_{j}}=\frac{d f}{d S} \frac{\partial S}{\partial x_{j}},
$$

and deriving, the transport equation for the apparent viscosity can be finally obtained

$$
\frac{\partial \mu_{a p p}}{\partial t}+u_{j} \frac{\partial \mu_{a p p}}{\partial x_{j}}=\frac{d f}{d S} \frac{\partial S}{\partial t}+\frac{d f}{d S} u_{j} \frac{\partial S}{\partial x_{j}}=\frac{d f}{d S}\left(\frac{\partial S}{\partial t}+u_{j} \frac{\partial S}{\partial x_{j}}\right) .
$$

The apparent viscosity must be multiplied for the transport equation, Eq. (25), in order to have a structure similar to the transport equation of $\$^{2}$. Let assume the apparent viscosity is function of the square of the shear rate, $S^{2}$. To avoid confusion between the second and the first derivatives with respect the square of a variable let assume 
$S^{2}=A$.

The apparent viscosity is then

$\mu_{a p p}=f\left(A\left(x_{j}, t\right)\right)$,

and, using the following relations

$\frac{\partial \mu_{a p p}}{\partial t}=\frac{d f}{d A} \frac{\partial S^{2}}{\partial t}, \frac{\partial \mu_{a p p}}{\partial x_{j}}=\frac{d f}{d A} \frac{\partial S^{2}}{\partial x_{j}}$,

the transport equation for apparent viscosity can be finally written as

$\frac{\partial \mu_{a p p}}{\partial t}+u_{j} \frac{\partial \mu_{a p p}}{\partial x_{j}}=\frac{d f}{d A} \frac{\partial S^{2}}{\partial t}+\frac{d f}{d A} u_{j} \frac{\partial S^{2}}{\partial x_{j}}=\frac{d f}{d A}\left(\frac{\partial S^{2}}{\partial t}+u_{j} \frac{\partial S^{2}}{\partial x_{j}}\right)$.

Defining

$C=\frac{d f}{d A}$,

and multiplying Eq. (23) by $2 C$, Eq. (32) can be written as

$\rho \frac{\partial \mu_{a p p}}{\partial t}+\rho u_{k} \frac{\partial \mu_{a p p}}{\partial x_{k}}=\frac{\partial}{\partial x_{k}}\left(\left(\mu_{a p p}+2 C S^{2}\right) \frac{\partial \mu_{a p p}}{\partial x_{k}}-2 C S_{k j} \frac{\partial p}{\partial x_{j}}\right)+$
$\left(\frac{\partial p}{\partial x_{j}}-\frac{\partial}{\partial x_{k}}\left(\mu_{a p p} S_{i j}\right)\right) \frac{\partial}{\partial x_{k}}\left(2 C S_{i j}\right)$

In conclusion, Eq. (34) is the transport equation of the apparent viscosity, which is a differential equation rather than an algebraic expression. The form of Eq. (34) is similar to the $\$^{2}$ transport equation.

\section{Transport equation for turbulent dissipation rate}

The turbulent dissipation rate is defined as

$\varepsilon=\frac{2}{\rho}\left\langle\mu_{a p p}\right\rangle\left\langle \$^{2}\right\rangle+\frac{2}{\rho}\left\langle\mu_{a p p}^{\prime} \$^{2}\right\rangle$

while its instantaneous component is

$\varepsilon^{\prime}=\frac{2}{\rho} \mu_{a p p} \$^{2}$

and its transport equation can be obtained by the sum of Eq. (25), multiplied by $4 \mu_{\text {app }} / \rho$, and Eq. (34), multiplied by $2 \$^{2} / \rho$. The instantaneous dissipation rate is finally averaged to give the detailed form of the transport equation

$\rho \frac{\partial \varepsilon}{\partial t}+\rho U_{k} \frac{\partial \varepsilon}{\partial x_{k}}=P^{\varepsilon}+\Pi^{\varepsilon}+T^{\varepsilon}+D^{\varepsilon}-\varepsilon^{\varepsilon}$,

where the groups of terms on the right hand side are given by 


$$
\begin{aligned}
& P^{\varepsilon}=\frac{2}{\rho}\left(\left\langle\mu_{a p p} \frac{\partial T_{R, i j}^{\prime}}{\partial x_{k}}\right\rangle \frac{\partial\left\langle S_{i j}\right\rangle}{\partial x_{k}}\right)-4\left\langle\mu_{a p p} \frac{\partial k^{\prime}}{\partial x_{k}}\right\rangle \frac{\partial\left\langle S_{k j}\right\rangle}{\partial x_{j}}+\frac{1}{\rho} \frac{\partial T_{\mu, i j}}{\partial x_{k}} \frac{\partial}{\partial x_{k}}\left(T_{R, i j}+T_{\mu, i j}+2\left\langle\mu_{a p p}\right\rangle\left\langle S_{i j}\right\rangle\right) \\
& -2 \frac{\partial T_{\mu, i j}}{\partial x_{k}} \frac{\partial k}{\partial x_{j}} \\
& \Pi^{\varepsilon}=\frac{\partial}{\partial x_{k}}\left(-\frac{4}{\rho}\left\langle\mu_{a p p} S_{k j}^{\prime} \frac{\partial p^{\prime}}{\partial x_{j}}\right\rangle-\frac{4}{\rho}\left\langle C \$^{2} S_{k j} \frac{\partial p}{\partial x_{j}}\right\rangle\right)_{, k}+\frac{4}{\rho}\left\langle\frac{\partial}{\partial x_{k}}\left(\mu_{a p p} S_{k j}^{\prime}\right) \frac{\partial p^{\prime}}{\partial x_{j}}\right\rangle+\frac{4}{\rho}\left\langle\frac{\partial}{\partial x_{k}}\left(C \$^{2} S_{k j}\right) \frac{\partial p}{\partial x_{j}}\right\rangle \\
& T^{\varepsilon}=\frac{\partial}{\partial x_{k}}\left(-\left\langle\rho u_{k}^{\prime} \varepsilon^{\prime}\right\rangle\right) \\
& \left.D^{\varepsilon}=\frac{\partial}{\partial x_{k}}\left(-\frac{1}{\rho} T_{\mu, i j} \frac{\partial}{\partial x_{k}}\left(T_{R, i j}+T_{\mu, i j}+2\left\langle\mu_{a p p}\right\rangle\left\langle S_{i j}\right\rangle\right)+2 T_{\mu, i j} \frac{\partial k}{\partial x_{j}}\right\rangle+2\left\langle\varepsilon^{\prime}\left(1+\frac{C S^{2}}{\mu_{a p p}}\right) \frac{\partial \mu_{a p p}}{\partial x_{k}}\right\rangle+\frac{4}{\rho}\left\langle\mu_{a p p} S_{i j}^{\prime} \frac{\partial}{\partial x_{k}}\left(\mu_{a p p}\left\langle S_{i j}\right\rangle\right)\right\rangle\right) \\
& \mathcal{E}^{\varepsilon}=\frac{4}{\rho}\left\langle\frac{\partial}{\partial x_{k}}\left(\mu_{a p p} S_{i j}\right) \frac{\partial}{\partial x_{k}}\left(\mu_{a p p} S_{i j}^{\prime}+C \$^{2} S_{i j}\right)\right\rangle
\end{aligned}
$$

\section{Discussion on the conservation equation for the dissipation rate.}

Each group of terms of Eq. (38) is discussed in the following.

The group $P^{\varepsilon}$ represents the production of dissipation rate which involves the instantaneous apparent viscosity, the instantaneous Reynolds stresses tensor, the mean rate of deformation tensor, the instantaneous turbulent kinetic energy, the gradient of the polymeric stresses tensor and the gradient of the total stresses tensor acting on the mean flow. In a Newtonian fluid the similar term is the product of the gradients of the Reynolds stresses tensor and the mean rate of deformation tensor multiplied the viscosity.

The three groups $\Pi^{\varepsilon}+T^{\varepsilon}+D^{\varepsilon}$ represent the transport of dissipation rate. The first group, $\Pi^{\varepsilon}$, is connected to the static pressure, respectively fluctuating and instantaneous, with the first one similar to a Newtonian fluid. The second group, $T^{\varepsilon}$, represents the turbulent transport due to vortices. The third group, $D^{\varepsilon}$, is made of five terms. The first two terms represent the turbulent transport due to apparent viscosity and molecular transport or average apparent viscosity, with the second term, involving molecular and turbulent transport due to viscosity fluctuations, present because of the viscosity variation with the position. The remaining three terms of the third group, $D^{\varepsilon}$, are completely new. The third term, which involves the mean rate of deformation tensor and the apparent viscosity, is similar to the transport of the turbulent kinetic energy term, where is present the product of the fluctuating viscosity, the tensors of the mean strain rate and the fluctuating velocity. The fourth term is made with the product of the polymeric stresses by the gradient of the total stresses acting on the mean flow. The fifth term is made with the product of the polymeric stresses by the gradient of the turbulent kinetic energy.

The group $\mathcal{E}^{\varepsilon}$ represents the dissipation contribution. The first term is a typical dissipation one made by the sum of a term similar to a Newtonian fluid while the last term is due to the equation for the transport of viscosity. 
Appendix A: Transport equation for the dissipation rate of turbulent kinetic energy

The step by step algebra to derive the equation for the dissipation rate is the following:

Multiplication of the transport equation for the rate of deformation tensor components, Eq. (25), by $4 \frac{\mu_{a p p}}{\rho}$

$\left.\left.\left.\frac{2}{\rho} \mu_{q p} \rho \frac{D \$^{2}}{D t}=\frac{2}{\rho} \mu_{q p p} \nabla\left[T_{R}^{\prime}\right]: \nabla[S]\right\rangle-4 \mu_{q p p} \nabla k^{\prime} \cdot(\nabla \cdot\langle S]\rangle\right)-\frac{4}{\rho} \mu_{q p} \nabla\left(\mu_{q p p}^{\prime}[S]\right\rangle\right): \nabla[S]-\frac{4}{\rho} \nabla \mu_{q p} \cdot[S]: \nabla\left(\mu_{q p p}^{\prime}\langle[S]\rangle\right)$

$\nabla \cdot\left(-\frac{4}{\rho} \mu_{q p p}[S] \nabla p^{\prime}-\rho \overrightarrow{u^{\prime}} \frac{2}{\rho} \mu_{q p p} \$^{2}+\mu_{q p} \frac{2}{\rho} \mu_{q p p} \nabla \$^{2}+\frac{4}{\rho} \mu_{q p p} \$^{2} \nabla \mu_{q p p}\right)+$

$\nabla \cdot\left(\frac{4}{\rho} \mu_{\text {qpp }}[S]: \nabla\left(\mu_{\text {qpp }}^{\prime}\langle S]\right\rangle\right)+4 \mu_{\text {qpp }}^{\prime}[S] \nabla k-\frac{2}{\rho} \mu_{\text {qpp }}^{\prime}[S]: \nabla\left(\left[T_{R}\right]+\left[T_{\mu}\right]\right)+4\left\langle\mu_{\text {qpp }}\right\rangle[S] \nabla k-\frac{2}{\rho}\left\langle\mu_{\text {qpp }}\right\rangle[S]: \nabla\left(\left[T_{R}\right]+\left[T_{\mu}\right]\right)+\cdot$

$\frac{4}{\rho} \nabla \mu_{q p p} \cdot[S] \nabla p^{\prime}+\frac{2}{\rho} \nabla \mu_{q p} \cdot \overrightarrow{\mu{ }^{\prime}} \$^{2}-\frac{2}{\rho} \nabla \mu_{q p p} \cdot \mu_{q p p} \nabla \$^{2}-\frac{4}{\rho} \nabla \mu_{q p} \cdot \$^{2} \nabla \mu_{q p}-4 \nabla \mu_{q p}^{\prime} \cdot[S] \nabla k+\frac{2}{\rho} \nabla \mu_{q p}^{\prime} \cdot[S]: \nabla\left(\left[T_{R}\right]+\left[T_{\mu}\right]\right)$

$\frac{4}{\rho} \mu_{\text {qp }} \nabla p^{\prime} \cdot\left(\nabla \cdot\left[S^{\prime}\right]\right)-\frac{4}{\rho} \mu_{\text {qqp }} \nabla\left(\mu_{\text {qp }}[S]\right): \nabla\left[S^{\prime}\right]+\frac{2}{\rho} \mu_{\text {qpp }} \nabla\left(\left[T_{R}\right]+\left[T_{\mu}\right]\right): \nabla\left[S^{\prime}\right]-4 \mu_{\text {qp }} \nabla k \cdot\left(\nabla \cdot\left[S^{\prime}\right]\right)+$

$-4 \nabla\left\langle\mu_{\text {qpp }}\right\rangle \cdot[S] \nabla k+\frac{2}{\rho} \nabla\left\langle\mu_{\text {qpp }}\right\rangle \cdot[S]: \nabla\left(\left[T_{R}\right]+\left[T_{\mu}\right]\right)$

Use the expression $\$^{2}=\left[S^{\prime}\right]:\left[S^{\prime}\right]$ to decompose some terms of Eq. (A.1)

$\left.\left.\left.\frac{2}{\rho} \mu_{q p} \rho \frac{D \$^{2}}{D}=\frac{2}{\rho} \mu_{q p} \nabla\left[T_{R}^{\prime}\right]: \nabla\langle S]\right\rangle-4 \mu_{q p} \nabla k^{\prime} \cdot(\nabla \cdot\langle S]\rangle\right) \frac{4}{\rho} \nabla\left(\mu_{q p}^{\prime}\langle S]\right\rangle\right): \nabla\left(\mu_{q p}[S]\right)+$

$\nabla \cdot\left(-\frac{4}{\rho} \mu_{q p p}[S] \nabla p^{\prime}-\rho \overrightarrow{u_{i}} \frac{2}{\rho} \mu_{q p} \$^{2}+\mu_{q p} \frac{2}{\rho} \mu_{q p} \nabla \$^{2}+\frac{4}{\rho} \mu_{q p} \$^{2} \nabla \mu_{q p}\right)+$

$\nabla \cdot\left(\frac{4}{\rho} \mu_{q p}[S]: \nabla\left(\mu_{q p}^{\prime}\langle S]\right\rangle\right)+2\left[T_{\mu}^{\prime}\right] \nabla k-\frac{1}{\rho}\left[T_{\mu}^{\prime}\right]: \nabla\left(\left[T_{R}\right]+\left[T_{\mu}\right]+4\left\langle\mu_{q p}\right\rangle[S] \nabla k-\frac{2}{\rho}\left\langle\mu_{q p}\right\rangle[S]: \nabla\left(\left[T_{R}\right]+\left[T_{\mu}\right]\right)+\right.$

$\frac{2}{\rho} \nabla \mu_{q p} \cdot \overrightarrow{\mu u}[S]:[S]-\frac{4}{\rho} \nabla\left(\mu_{q p}[S]\right): \nabla\left(\mu_{q p}[S]\right)+\frac{4}{\rho} \nabla p^{\prime} \cdot\left(\nabla \cdot \mu_{q p}[S]\right)+\frac{1}{\rho} \nabla\left(\left[T_{R}\right]+\left[T_{\mu}\right] \vdots: \nabla\left[T_{\mu}^{\prime}\right]-2 \nabla k \cdot\left(\nabla \cdot\left[T_{\mu}^{\prime}\right]+\right.\right.$

$-4\left(\nabla \cdot\left\langle\mu_{q p}\right\rangle[S]\right) \cdot \nabla k+\frac{2}{\rho} \nabla\left(\left\langle\mu_{q p}\right\rangle[S]\right): \nabla\left(\left[T_{R}\right]+\left[T_{\mu}\right]\right.$

The instantaneous viscosity $\mu_{a p p}$ needs to be summed to the turbulent one $\$$. The symbol $\frac{D}{D t}$ is used for the total derivative in the conservation equation of the apparent viscosity, recalling the relation

$\frac{D}{D t}=\frac{D}{D t}+\vec{u}^{\prime} \cdot \nabla$

It is then possible to write

$$
\begin{aligned}
& 2 \frac{\$^{2}}{\rho} \rho \frac{\partial \mu_{a p p}}{\partial t}+2 \frac{\$^{2}}{\rho} \rho \vec{U} \cdot \nabla \mu_{a p p}+2 \frac{\$^{2}}{\rho} \rho \vec{u}^{\prime} \cdot \nabla \mu_{a p p}= \\
& \nabla \cdot\left(-4 \frac{\$^{2}}{\rho} C[S] \nabla p+2 \mu_{a p p} \frac{\$^{2}}{\rho} \nabla \mu_{a p p}+4 \frac{\$^{2}}{\rho} C S^{2} \nabla \mu_{a p p}\right)+\frac{4}{\rho} \nabla\left(C \$^{2}\right) \cdot([S] \nabla p) \\
& -\frac{1}{C} \nabla\left(2 C \frac{\$^{2}}{\rho}\right) \cdot\left(\mu_{q p p} \nabla \mu_{a p p}\right)-\frac{4}{\rho} \nabla\left(C \$^{2}\right) \cdot\left(S^{2} \nabla \mu_{a p p}\right)+4 \frac{\$^{2}}{\rho} C(\nabla \cdot[S]) \cdot \nabla p-4 \frac{\$^{2}}{\rho} C \nabla\left(\mu_{q p p}[S]\right): \nabla[S]
\end{aligned}
$$

Collection of the different terms gives 


$$
\begin{aligned}
& 2 \frac{\$^{2}}{\rho} \rho \frac{D \mu_{a p p}}{D t}=\nabla \cdot\left(-\frac{4}{\rho} C \$^{2}[S] \nabla p+2 \mu_{a p p} \frac{\$^{2}}{\rho} \nabla \mu_{a p p}+\frac{4}{\rho} \$^{2} C S^{2} \nabla \mu_{a p p}\right)+ \\
& \nabla \cdot\left(\frac{4}{\rho} C \$^{2}[S]\right) \cdot \nabla p-\frac{4}{\rho} \nabla\left(\mu_{a p p}[S]\right): \nabla\left(C \$^{2}[S]\right)-\frac{2}{\rho} \nabla \mu_{a p p} \cdot \rho \vec{u}^{\prime}\left[S^{\prime}\right]:\left[S^{\prime}\right]
\end{aligned} .
$$

Summation of Eq. (A.2) and Eq. (A.5) gives

$$
\begin{aligned}
& \left.\left.\frac{2}{\rho} \mu_{q p p} \rho \frac{D \$^{2}}{D t}+\frac{2}{\rho} \$^{2} \rho \frac{D \mu_{q p p}}{D t}=\frac{2}{\rho} \mu_{q p p} \nabla\left[T_{R}^{\prime}\right]: \nabla[S]\right\rangle-4 \mu_{q p p} \nabla k^{\prime} \cdot(\nabla \cdot\langle S]\rangle\right) \\
& \nabla \cdot\left(-\overrightarrow{\rho \prime} \frac{2}{\rho} \mu_{\text {qqp }} \$^{2}+\mu_{\text {qpp }} \frac{2}{\rho} \mu_{\text {qpp }} \nabla \$^{2}+\mu_{\text {qpp }} \frac{2}{\rho} \$^{2} \nabla \mu_{\text {qpp }}+\frac{4}{\rho} \mu_{\text {qqp }} \$^{2} \nabla \mu_{\text {qpp }}+\frac{4}{\rho} \$^{2} C S^{2} \nabla \mu_{\text {qpp }}\right)+ \\
& \nabla \cdot\left(\frac{4}{\rho} \mu_{\text {qpp }}[S]: \nabla\left(\mu_{\text {qp }}^{\prime}\langle S]\right\rangle\right)+2\left[T_{\mu}^{\prime}\right] \nabla k-\frac{1}{\rho}\left[T_{\mu}^{\prime}\right]: \nabla\left(\left[T_{R}\right]+\left[T_{\mu}\right]\right)+4\left\langle\mu_{\text {qpp }}\right\rangle[S] \nabla k-\frac{2}{\rho}\left\langle\mu_{\text {qpp }}\right\rangle[S]: \nabla\left(\left[T_{R}\right]+\left[T_{\mu}\right]\right)+. \\
& \nabla \cdot\left(\frac{4}{\rho} \mu_{\text {qpp }}[S] \nabla p^{\prime}-\frac{4}{\rho} C S^{2}[S] \nabla p\right)-\frac{4}{\rho} \nabla\left(\mu_{\text {qpp }}[S]\right): \nabla\left(\mu_{\text {qpp }}[S]\right)-\frac{4}{\rho} \nabla\left(\mu_{q p p}[S]\right): \nabla\left(C \$^{2}[S]\right)+ \\
& \left.\frac{4}{\rho} \nabla\left(\mu_{q p}^{\prime}[S]\right\rangle\right): \nabla\left(\mu_{q p p}[S]\right)+\frac{4}{\rho} \nabla p^{\prime} \cdot\left(\nabla \cdot \mu_{q p p}[S]\right)+\nabla \cdot\left(\frac{4}{\rho} C S^{2}[S]\right) \cdot \nabla p+ \\
& \frac{1}{\rho} \nabla\left(\left[T_{R}\right]+\left[T_{\mu}\right]\right): \nabla\left[T_{\mu}^{\prime}\right]-2 \nabla k \cdot\left(\nabla \cdot\left[T_{\mu}^{\prime}\right]\right)-4\left(\nabla \cdot\left\langle\mu_{\text {qpp }}\right\rangle[S]\right) \cdot \nabla k+\frac{2}{\rho} \nabla\left(\left\langle\mu_{\text {qpp }}\right\rangle[S]\right): \nabla\left(\left[T_{R}\right]+\left[T_{\mu}\right]\right)
\end{aligned}
$$

Introduction of the instantaneous dissipation rate $\frac{2}{\rho} \mu_{a p p} \$^{2}=\mathcal{E}^{\prime}$, multiplication and division for $\mathrm{C}$ of the apparent viscosity gives

$$
\begin{aligned}
& \left.\left.\rho \frac{D \varepsilon^{\prime}}{D}=\frac{2}{\rho} \mu_{q p} \nabla\left[T_{R}^{\prime}\right]: \nabla\langle S]\right\rangle-4 \mu_{q p} \nabla k^{\prime} \cdot(\nabla \cdot\langle S]\rangle\right)+\frac{1}{\rho} \nabla\left(\left[T_{R}\right]+\left[T_{\mu}\right] \vdots \nabla\left[T_{\mu}^{\prime}\right]-2 \nabla k \cdot\left(\nabla \cdot\left[T_{\mu}^{\prime}\right]+\right.\right.
\end{aligned}
$$

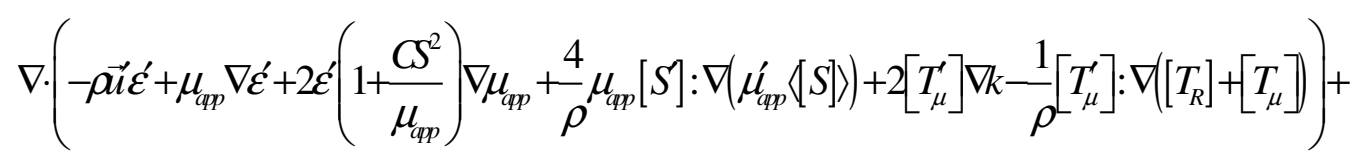

$$
\begin{aligned}
& \nabla \cdot\left(\frac{4}{\rho} \mu_{q p}[S] \nabla p^{\prime}-\frac{4}{\rho} C S^{2}[S] \nabla p+4\left\langle\mu_{q p}\right\rangle[S] \nabla k-\frac{2}{\rho}\left\langle\mu_{q p p}\right\rangle[S]: \nabla\left(\left[T_{R}\right]+\left[T_{\mu}\right]\right)+\frac{4}{\rho} \nabla p^{\prime} \cdot\left(\nabla \cdot \mu_{q p}[S]\right)+\right.\text {. } \\
& \left.\nabla \cdot\left(\frac{4}{\rho} C S^{2}[S]\right) \cdot \nabla p-\frac{4}{\rho} \nabla\left(\mu_{q p}[S]\right): \nabla\left(\mu_{q p p}[S]\right)-\frac{4}{\rho} \nabla\left(\mu_{q p p}[S]\right): \nabla\left(C S^{2}[D]\right)-\frac{4}{\rho} \nabla\left(\mu_{q p p}[S]\right\rangle\right): \nabla\left(\mu_{q p p}[S]\right)+ \\
& -4\left(\nabla \cdot\left\langle\mu_{q p}\right\rangle[S]\right) \cdot \nabla k+\frac{2}{\rho} \nabla\left(\left\langle\mu_{q p p}\right\rangle[S]\right): \nabla\left(\left[T_{R}\right]+\left[T_{\mu}\right]\right.
\end{aligned}
$$

Further algebra is necessary for the dissipation terms

$$
\begin{aligned}
& \rho \frac{D \epsilon^{\prime}}{D}=\frac{2}{\rho} \mu_{q p} \nabla\left[T_{R}^{\prime}\right]: \nabla\langle S \mid\rangle-4 \mu_{q p} \nabla k^{\prime} \cdot(\nabla \cdot\langle S \mid\rangle)+\frac{1}{\rho} \nabla\left[T_{\mu}^{\prime}\right]: \nabla\left(\left[T_{R}\right]+\left[T_{\mu}\right]+2\left\langle\mu_{q p}\right\rangle\langle S \mid\rangle\right)-2 \nabla k \cdot\left(\nabla \cdot\left[T_{\mu}^{\prime}\right]\right) \\
& \nabla \cdot\left(-\vec{\mu} \dot{\varepsilon}^{\prime}+\mu_{\varphi p} \nabla \dot{\varepsilon}^{\prime}+2 \dot{\varepsilon}^{\prime}\left(1+\frac{C^{2}}{\mu_{q p}}\right) \nabla \mu_{q p}+\frac{4}{\rho} \mu_{\mu p}[S]: \nabla\left(\mu_{\varphi p}\langle S \mid\rangle\right)+2\left[T_{\mu}^{\prime}\right] \nabla k-\frac{1}{\rho^{L}}\left[T_{\mu}^{\prime}\right]: \nabla\left(\left[T_{R}\right]+\left[T_{\mu}\right]+2\left\langle\mu_{q p}\right\rangle\langle S]\right\rangle\right)+ \\
& \left.\nabla \cdot\left(4\left\langle\mu_{q p}\right\rangle[S] \nabla k-\frac{2}{\rho}\left\langle\mu_{q p}\right\rangle[S]: \nabla\left(\left[T_{R}\right]+\left[T_{\mu}\right]+2\left\langle\mu_{q p}\right\rangle[S]\right\rangle\right)-\frac{4}{\rho} \mu_{q p}[S] \nabla p^{\prime}-\frac{4}{\rho} C S^{2}[S] \nabla p\right)+\frac{4}{\rho} \nabla p^{\prime} \cdot\left(\nabla \cdot \mu_{q p}[S]\right)+ \\
& \left.\left.\nabla \cdot\left(\frac{4}{\rho} C S^{2}[S]\right) \cdot \nabla p-\frac{4}{\rho} \nabla\left(\mu_{q p}[S]\right): \nabla\left(\mu_{q p}[S]+C S^{2}[S]\right)-4\left(\nabla \cdot\left\langle\mu_{q p}\right\rangle[S]\right) \cdot \nabla k+\frac{2}{\rho} \nabla\left(\left\langle\mu_{q p}\right\rangle[S]\right): \nabla\left(\left[T_{R}\right]+\left[T_{\mu}\right]+2\left\langle\mu_{q p}\right\rangle S S\right]\right\rangle\right)
\end{aligned}
$$


Averaging the previous equation allows obtaining the dissipation rate equation as Eq. (40).

\section{Conclusions}

The mass conservation equation is the same as for a Newtonian fluid because is not dependent on the fluid, while the momentum conservation equation is different because of the presence of the polymeric stresses.

The turbulent kinetic energy and dissipation rate equations contain terms proportional to the viscosity of a Newtonian fluid while, for a GNF, they are proportional to the mean product of the instantaneous variables. Further on, the equations for a non-Newtonian fluid contain terms with viscosity fluctuations and derivatives of the viscosity.

In conclusion, the exact equations derived for a GNF can reduce to those for a Newtonian fluid if the apparent viscosity fluctuations and the $\mathrm{C}$ coefficient disappear and the mean apparent viscosity is constant. An important conclusion of the present paper is the derivation of the dissipation rate in the conservative form of Eq. (20).

\section{References}

[1] F. Gori, Effects of Variable Physical Properties in Laminar Flow of Pseudo-plastic Fluids, International Journal of Heat and Mass Transfer, 21(2), (1978) 247-250.

[2] F. Gori, Variable Physical Properties in Laminar Heating of Pseudo-plastic Fluids with Constant Wall Heat Flux, Journal of Heat Transfer, ASME, 100(2), (1978) 220-223.

[3] M. Rudman, H.M. Blackburn, Direct numerical simulation of turbulent non-Newtonian flow using a spectral element method, Applied Mathematical Modelling, 30 (2006) 1229-1248.

[4] M. Rudman, H.M. Blackburn, L.J.W. Graham, L. Pullum, Turbulent pipe flow of shear-thinning fluids, J. Non-Newtonian Fluid Mech., 118 (2004) 33-48.

[5] C. D. Dimitropoulos, R. Sureshkumar, A. N. Beris, Direct numerical simulation of viscoelastic turbulent channel flow exhibiting drag reduction: effect of the variation of rheological parameters, J. Non-Newtonian Fluid Mech., 79 (1998) 433-468.

[6] A. N. Beris, C. D. Dimitropoulos, Pseudo-spectral simulation of turbulent viscoelastic channel flow, Comp. Methods Appl. Mech. Eng., 180 (1990) 365-392.

[7] R. Benzi, I. Procaccia, Simple model for drag reduction, Physical Review E, 68 (2003) 025303(R).

[8] S.M. Son, R.K. Singh, Turbulence modelling and verification for aseptically processed soybean milk under turbulent flow conditions, Journal of Food Engineering, 52 (2002) 177-184.

[9] W. Binxin, C. Shulin, CFD Simulation of Non-Newtonian Fluid Flow in Anaerobic Digesters Biotechnology and Bioengineering, Biotechnology and Bioengineering, 99(3), (2008) 15.

[10] M. R. Malin, Turbulent pipe flow of power-law fluids, Int. Comm. Heat Mass Transfer, 24(7) (1997) 977-988.

[11] M. R. Malin, Turbulent pipe flow of Herschel-Bulkley fluids, Int. Comm. Heat Mass Transfer, 25(3), (1998) 321-330. 
[12] P.J. Oliveira, F.T. Pinho, A qualitative assessment of the role of a viscosity depending on the third invariant of the rate-of-deformation tensor upon turbulent non-Newtonian flow, J. Non-Newtonian Fluid Mech., 78 (1998) 1-25.

[13] F. T. Pinho, A GNF framework for turbulent flow models of drag reducing fluids and proposal for a $k-$ $\varepsilon$ type closure, J. Non-Newtonian Fluid Mech., 114 (2003) 149-184.

[14] C.F. Li, V.K. Gupta, R. Sureshkumar, B. Khomami, Turbulent channel flow of dilute polymeric solutions: Drag reduction scaling and an eddy viscosity model, J. Non-Newtonian Fluid Mech., 139 (2006) 177-189.

[15] F.T. Pinho, C.F. Li, B.A. Younis, R. Sureshkumar, A low Reynolds number turbulence closure for viscoelastic fluids, J. Non-Newtonian Fluid Mech., 154 (2008) 89-108.

[16] G.K. Batchelor, Computation of the energy spectrum in homogeneous two-dimensional turbulence, Physics of Fluids, II (1969) 233-9.

[17] R.H. Kraichnan, D. Montgomery, Two-dimensional turbulence. Reports on Progress in Physics, 43(5), (1980) 547-619.

[18] R.H. Kraichnan, Eddy viscosity in two and three dimensions, Journal of the Atmospheric Sciences, 33(8), (1976) 1521-36.

[19] R.H. Kraichnan, Statistical dynamics of two-dimensional flow, Journal of Fluid Mechanics, 67(1), (1975) 155-75.

[20] J. R. Herring, S. A. Orszag, R. H. Kraichnan, D. G. Fox, Decay of Two-Dimensional Homogeneous Turbulence, Journal of Fluid Mechanics, 66(3), (1974) 417-444.

[21] P.A. Davidson, Turbulence an introduction for scientist and Engineers, Oxford University Press, 2004.

[22] H. Tennekes, J. L. Lumley, A First Course in Turbulence, MIT Press, Cambridge, MA, 1972.

[23] D.O.A. Cruz, F.T. Pinho, P.R. Resende, Modeling the new stress for improved drag reduction predictions of viscoelastic pipe flow, J. Non-Newtonian Fluid Mech., 121 (2004) 127-141. 